\title{
Mucus clearance as a primary innate defense mechanism for mammalian airways
}

\author{
Michael R. Knowles and Richard C. Boucher \\ Cystic Fibrosis/Pulmonary Research and Treatment Center, The University of North Carolina, Chapel Hill, North Carolina, USA \\ Address correspondence to: Richard C. Boucher, Cystic Fibrosis/Pulmonary Research and Treatment Center, \\ 7011 Thurston-Bowles Building, CB\# 7248, The University of North Carolina, Chapel Hill, North Carolina 27599-7248, USA. \\ Phone: (919) 966-1077; Fax: (919) 966-7524; E-mail: rboucher@med.unc.edu.
}

J. Clin. Invest. 109:571-577 (2002). DOI:10.1172/JCI200215217.

The conducting airways branch $20-25$ times between the trachea and the alveoli as inhaled air passes from the relatively constricted nasal/tracheal passages to the large surface area of alveoli $\left(70 \mathrm{~m}^{2}\right)$, where gas exchange occurs. This branching anatomy leads to a surface area that expands greatly from proximal airways (e.g., third generation; $\sim 50 \mathrm{~cm}^{2}$ ) to distal airways (20th to 25 th generation; $\left.\sim 2 \mathrm{~m}^{2}\right)$. The regional differences in airway surface area (or airway perimeters; ref. 1), which is often depicted by showing the airways as an inverted funnel (Figure 1), pose interesting challenges for lung defense. Because many of the particles that settle on airway surfaces are infectious, airways have evolved innate defense mechanisms that constantly protect airways against bacterial and other types of infection.

There is still little agreement on the nature of these innate airway defense mechanisms $(2,3)$ (Figure 1). In the more traditional view, mechanical clearance of mucus is considered the primary innate airway defense mechanism (4-6). In this view, the role of the epithelia lining airway surfaces is to provide the integrated activities required for mucus transport, including ciliary activity and regulation of the proper quantity of salt and water on airway surfaces via transepithelial ion transport. More recently, a second view of innate airway defense has emerged as a result of studies of the pathogenesis of cystic fibrosis (CF) (7). This view emphasizes a role for a "chemical shield" in protecting the lung against inhaled bacteria (8). In this hypothesis, the two important functions for epithelia are the production of salt-sensitive defensins that are secreted into airway lumens, and the production of a low-salt $(<50 \mathrm{mM} \mathrm{NaCl})$ liquid on airway surfaces that renders defensins active (9).

The predictions of each of these models and the relevant data have been extensively reviewed $(2,3,10,11)$. Here, we will focus on the role of mucus clearance in the lung as the more important innate defense mechanism in health and disease, including CF. We will attempt to fill in the gaps in our knowledge regarding important aspects of the mucus clearance system, and, where relevant, point out differences between the two views of innate airway defense.

\section{Microanatomy of the airway surface}

With the advent of the capacity to fix airway surface liquid (ASL) in vivo, using the perfluorocarbon/osmium technique pioneered by Sims et al. (12), and the development of well-differentiated (WD) human airway epithelial cultures that exhibit mucus transport in vitro (13), it is now possible to investigate the microanatomy of mucus transport on airway surfaces at high resolution. Representative images depicting the range of morphologic techniques that can be applied to this culture system are shown in Figure 2.

Analysis of photomicrographs of this preparation, combined with immunocytochemical studies, have revealed several key features of the microanatomy of the ASL compartment $(13,14)$. The ASL consists of at least two layers, a mucus layer and a periciliary liquid layer (PCL; Figure 2). The mucus layer consists of high-molecular weight, heavily glycosylated macromolecules, products of at least two distinct genes (MUC5AC and $M U C 5 B)$, that behave as a tangled network of polymers

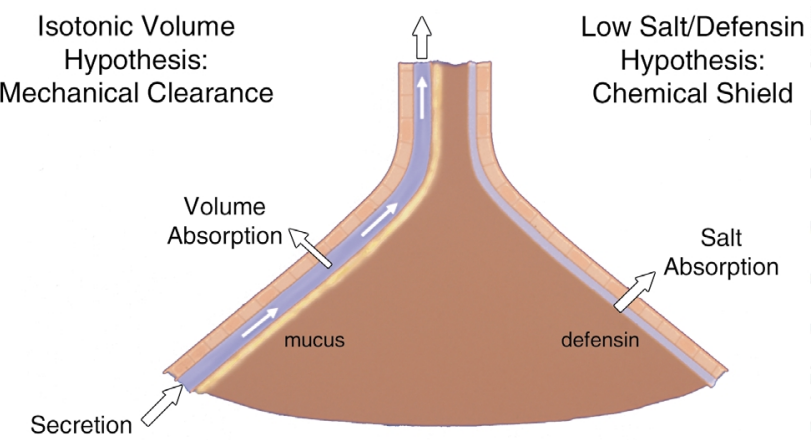

Figure 1

Pulmonary defense mechanisms preventing chronic bacterial infection. The lung is depicted as an inverted funnel, reflecting the relative surface area of distal versus proximal airways. The mechanical-clearance-of-mucus hypothesis is shown on the left. The schema depicts discrete mucus and periciliary liquid layers and ascribes to the epithelium a volume-absorbing function. The chemical shield hypothesis is shown on the right, with the epithelium depicted as having a salt- but not a volume-absorbing function to produce the hypotonic ASL required for defensin activity. 

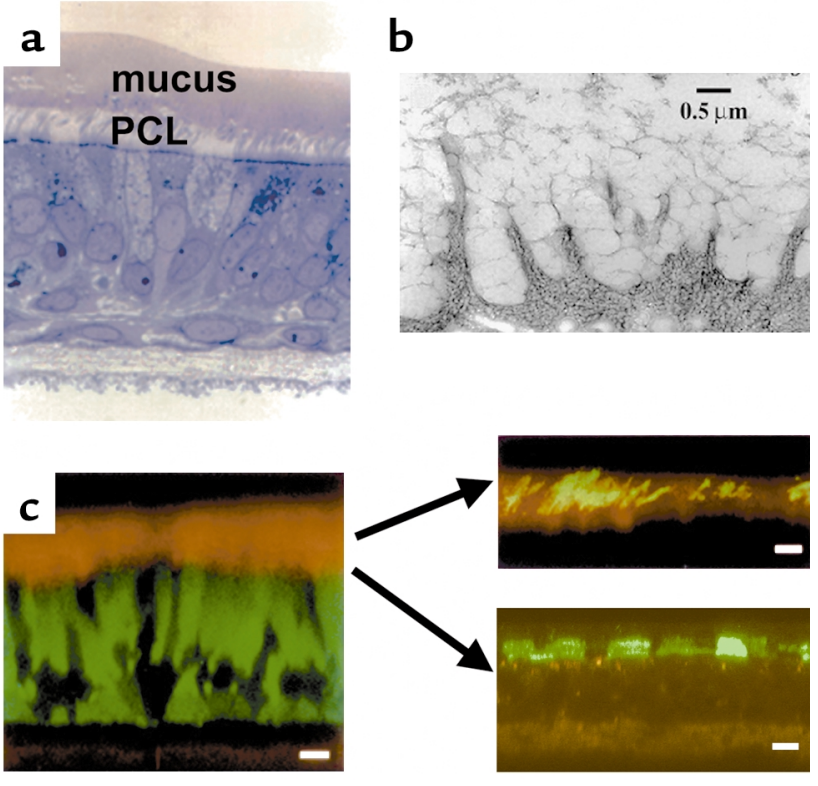

Figure 2

Microanatomy of human ASL. (a) WD human airway epithelial culture exhibiting rotational mucus transport (see Figure 3), fixed with perfluorocarbon/osmium. Note a distinct mucus layer atop a distinct PCL. (b) Visualization of glycocalyx on WD human airway epithelia by the freeze substitution technique. Note the high degree of organization of this barrier. (c) Left: X-Z confocal image of living WD human airway epithelial culture. The cells were stained with calcein, AM, (green), and the ASL was visualized with Texas red dextran. Scale bar $=10 \mu \mathrm{m}$. Top right: Fluorescent "dissection" of mucus layer and PCL in living WD airway epithelia by confocal microscopy. The mucus layer is visualized as green fluorescent beads and the PCL as the "bead-free zone" interposed between the mucus layer and cell surface (black). Scale bar $=10 \mu \mathrm{m}$. Bottom right: Detection of glycocalyx by fluorescence/confocal microscopy. The keratan sulfate component of the glycocalyx is visualized by Texas red-labeled anti-keratan sulfate (anti-KS) antibodies. Scale bar $=5 \mu \mathrm{m}$.

(15). The properties of the mucin gel are determined by this tangled network, which reflects, in part, the water content, monovalent and divalent ion concentrations, and $\mathrm{pH}$ of the ASL. It appears that mucin macromolecules are well adapted to binding and trapping inhaled particles for clearance from the lung, at least in part because of the extraordinary diversity of their carbohydrate side chains. Because they provide, in effect, a combinatorial library of carbohydrate sequences, mucins can bind to virtually all particles that land on airway epithelia and can thus clear them from the lung (16). It is still not known whether the mucus layer is continuous or discontinuous in human airways in vivo, although it appears likely at least that the layer is not homogeneous but is deeper at some sites than at others.

The mucus-free zone at the cell surface, which approximates the height of the outstretched cilia, has been previously described as the "sol" layer but is perhaps better termed the "periciliary liquid layer" (PCL). The ability to visualize this layer (Figure 2), both with perfluorocarbon/osmium/transmission electron microscopy fixation techniques and with confocal microscopy (Figure 3 ), has led to an appreciation of its vital importance in lung defense. As discussed below, this liquid layer is crucial both because it provides a low-viscosity solution in which cilia can beat rapidly (about $8-15 \mathrm{~Hz}$ ) and because it shields the epithelial cell surface from the overlying mucus layer.

One of the more important aspects of recent studies of epithelial cell surfaces and their relationship to mucus clearance has been the delineation of the size, composition, and functional aspects of the "glycocalyx" (Figure 2). A combination of more traditional freeze substitution and ruthenium red staining with new antibodies to cell surface-tethered mucins has revealed that the apical surfaces of airway epithelial cells are lined by highly glycosylated molecules that extend $500-1500 \mathrm{~nm}$ into the lumen $(17,18)$. These data have set the stage for studies that examine interactions in airway diseases between the mobile, gel-forming mucus layer, consisting of MUC5AC and MUC5B, and the cell surface-tethered mucins, such as MUC1 and MUC4.

\section{Physiology of mucus clearance from the lung}

Macroscopic transport of ASL. A number of techniques have been used to demonstrate that particles deposited on mammalian airway surfaces are cleared over relatively short periods of time $(4,19)$. A useful approach to quantitating basal mucus clearance in humans is to measure the clearance of inhaled and deposited radiolabeled particles from the human lung as a function of time with external scintography $(4,20)$. The basal rate of particle clearance depends on the interactions of cilia with the overlying mucus. It is not yet clear what determines the basal rates of mucociliary clearance, which vary within proximal and distal regions of the airways. Certainly, the ciliary beat frequency and effectiveness of cilia are primary determinants of the basal mucociliary clearance rate, but the quantity and viscoelastic properties of mucin, and possibly the viscous properties of the PCL, may also be important variables.

Studies of patients with airway diseases and analyses of the daily variability of clearance rates in normal human subjects reveal a wide range of mucociliary clearance rates, implying endogenous regulation of this process (4). It is not clear what mechanisms regulate mucociliary clearance rates in response to airway stresses. There is no cholinergic efferent innervation of the superficial airway epithelium (21), and it is likely that tachykinin-mediated neural systems are present predominantly in the upper airways, making it unlikely that neural mechanisms regulate lung mucus transport (21). Similarly, systemic hormonal responses, for example, to epinephrine, have time constants that are too long for this function. There has been interest in autocrine and paracrine airway epithelial signals that may control basal and stimulated rates of mucus clearance in the airways. The recognition that $5^{\prime}$ nucleotides applied to airway surfaces regulate all components of the mucus clearance system, including epithelial ion transport, ciliary beat frequency, and mucus secretion, has suggested a role for these compounds in this function (22-26). These speculations have been buttressed by recent data suggesting that shear stress induces 
nucleotide release in airway epithelia and that the airways express a large number of extracellular nucleotidases that regulate extracellular nucleotide concentrations (27-30). Collectively, the data suggest that nucleotide release, perhaps in response to ambient and cough-induced shear stress, regulates mucus clearance rates in response to luminal stresses.

It should be emphasized that the lungs possess an additional mechanism to clear mucus from the lung, namely cough clearance. By definition, cough clearance is independent of the actions of cilia, but cough efficiency is heavily dependent on several variables pertinent to ASL (31). The overall height and volume of liquid on the airway surface are directly related to the efficiency of cough clearance, whereas the viscosity of the luminal material is inversely related to the efficiency. As highlighted previously by Zahm and colleagues, the PCL appears to be an extraordinarily important contributor to the efficiency of cough clearance (32). Specifically, the lubricating function of the PCL facilitates mucus movement along airway surfaces in response to coughing. As discussed below, the absence of the PCL allows adhesive interactions between mobile mucins in the mucus layer and tethered cell-surface mucins, greatly reducing the efficiency of cough clearance.

Microscopic physiology of ASL/mucus transport. The development of cell culture methods in which WD lung epithelia expressing rotational mucus transport are examined using confocal microscopy to study the movement of the individual components of the mucus layer and PCL has provided great insight into the physiology of ASL clearance (Figure 3). Recent studies have demonstrated complex interactions among cilia, the mobile mucins in the mucus layer, and the underlying PCL (13, 14). For example, it appears that both the mucus layer and the PCL are moved unidirectionally along airway surfaces via ciliary actions from the small airways to the proximal airways and larynx (13). Interestingly, however, recent data have suggested that ciliary actions also impart vertical movements within the mucus layer (33). Thus, particles deposited on the surface of the mucus layer are rapidly mixed within the mucus layer. This feature of the mucus layer, i.e., entrapment, may constitute a second mechanism that ensures clearance of virtually any particle that deposits on airway surfaces. These studies also showed for the first time that the PCL moves with the mucus layer, elucidating a mechanism that clears the lung of hydrophilic noxious solutes dissolved in the PCL, along with particles trapped in mucus.

From a mechanistic point of view, these studies suggested that the movement of ASL on airway surfaces involved two steps. First, the ciliary power stroke acts on the undersurface of mucus to move the mucus layer unidirectionally on the airway surface. Second, the frictional interaction of the mucus layer with the PCL allows this underlying layer to travel at velocities similar to those of the overlying mucus layer (a form of "secondary" transport). These data are consistent with the fact that mammalian airway cilia are too short, and their recovery stroke not sufficiently close to the cell surface, to move the PCL directly. a

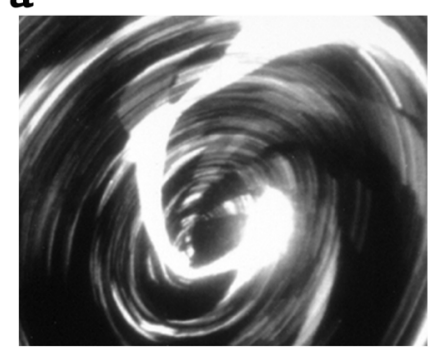

b
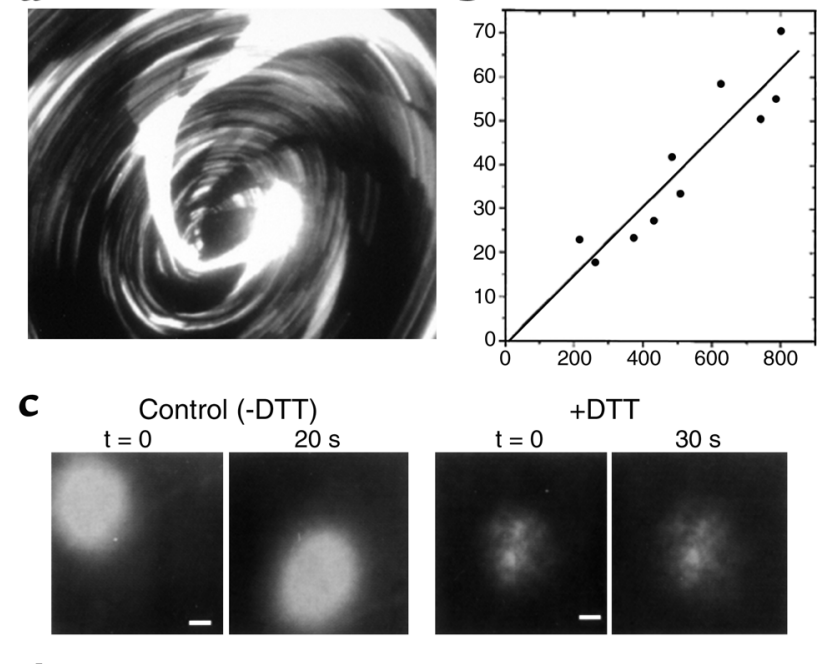

d
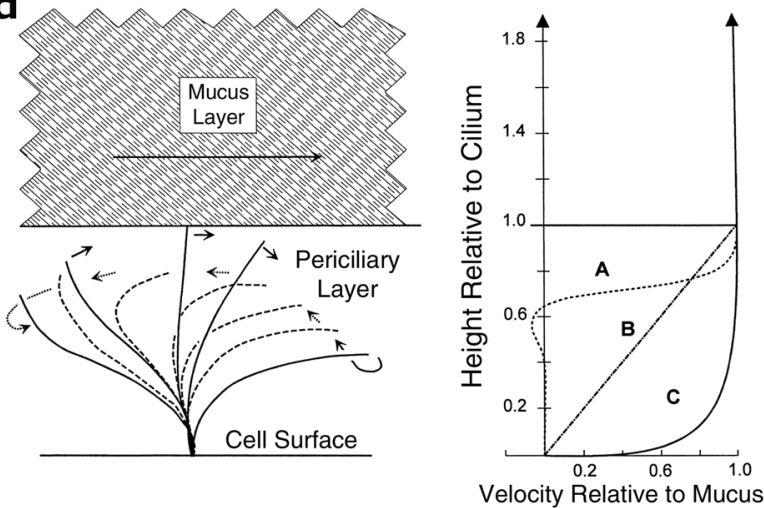

Figure 3

Mucus layer and PCL transport by human airway epithelia. (a) Mucus transport in WD airway epithelial cultures was identified by the rotational movement of $1-\mu \mathrm{m}$ fluorescent microspheres in the mucus layer. The image shown was acquired as a single 5 -second exposure; the streaks represent the paths of individual microspheres (field diameter $\sim 2 \mathrm{~mm}$ ). (b) Mucus transport rates were calculated as the linear velocities of fluorescent microspheres (a) and plotted against the distance from the center of the rotation (radial). (c) Transport of PCL revealed by photoactivation of caged fluorescein-conjugated dextrans. The ASL was labeled with caged fluorescein-conjugated dextran $(10,000 \mathrm{Da})$ and the fate of photoreleased dextran fluorescence was determined. Conventional fluorescence microscopy at low power was used (bars $=0.2 \mathrm{~mm}$ ). Left: Migration of the released fluorescent dextran during the 20-second period of observation. Right: After removing the mucus layer (+DTT), the migration of released fluorescent dextran was minimal. Both the mucus layer and the $\mathrm{PCL}$ are labeled by photoactivation. The absence of a "smear" during movement implies that both the mucus layer and the PCL move at the same velocity. The absence of PCL movement after mucus removal suggests that mucus movement is critical for PCL movement. (d) Models of ASL transport. Lateral fluid velocity profiles in ASL predicted for three different considerations, with the ordinate aligned to the diagram of the ciliary beat cycle at the left. Curve A approximates velocity profiles in the PCL predicted from theoretical considerations of ciliary propulsion of water $(60,61)$. Note nominally zero velocities at $70-75 \%$ of the ciliary length, below the level of the return stroke. Curve B depicts the velocity profile predicted for $\mathrm{PCL}$ flow driven solely by frictional interactions with the mucus layer. Curve $\mathrm{C}$ depicts the velocity profile for the PCL from the observations in this work that the flows of the PCL and mucus layer are essentially indistinguishable. 


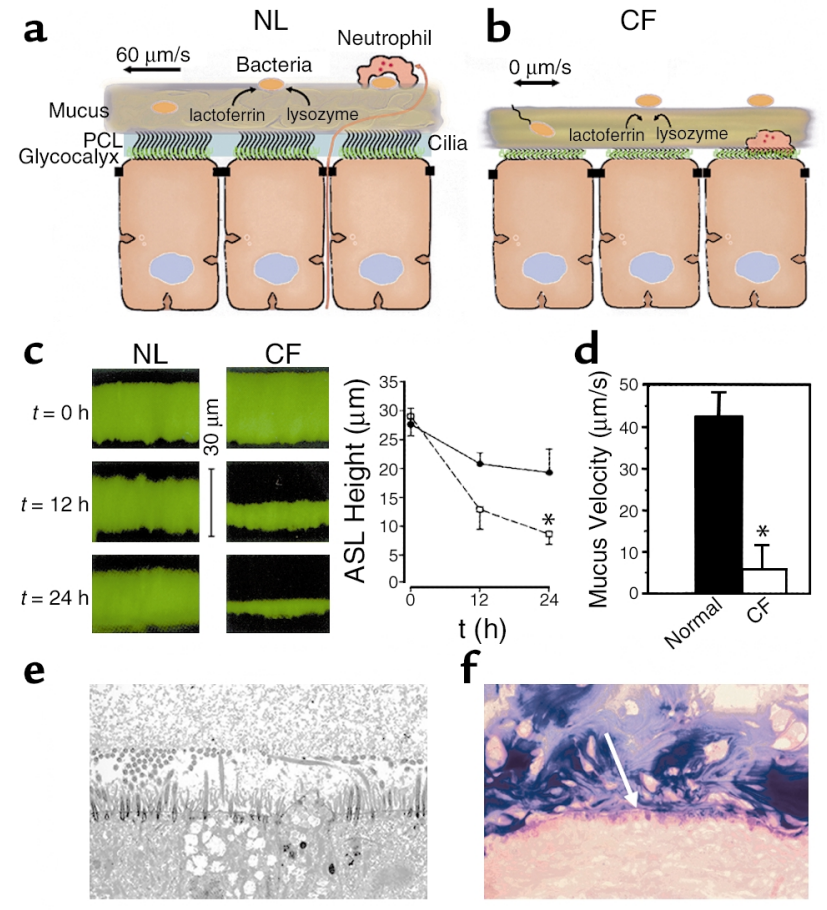

Figure 4

$P C L$ is required for effective mucociliary and cough clearance. (a) Schema of microanatomy of normal ASL. Note mixing of bacteria in "turbulent" mucus. (b) Schema depicting hypothetical volume depletion of ASL covering CF airway epithelial surfaces. Note that the volume depletion is reflected in both the generation of a more concentrated mucus layer and the depletion of the PCL. PCL depletion allows interactions to occur between the tethered mucins of the glycocalyx and the mucus layer. Note motile bacteria penetrating into thickened, stationary mucus. (c) Evidence for ASL volume depletion in CF airway epithelia. ASL height was measured immediately, 12 hours, and 24 hours after deposition of PBS containing Texas red dextran on the epithelial surface of the cell (pseudocolored green). Left: Representative confocal microscopy images. Right: Mean data for normal (circles) and CF (squares) ASL heights. ${ }^{*}$ CF ASL is significantly shallower than normal $(P<0.05 ; n=6$ per group). (d) Mucus (bead) rotational velocity 24 hours after administration of PBS containing fluorescent markers. At $t=0$ hours, both normal and CF cultures exhibited rotational velocities of about $45 \mu \mathrm{m} / \mathrm{s}$. $\left({ }^{*} P<0.05\right.$, CF vs. normal; $n=6$ per group). (e) Low-power electron micrograph of perfluorocarbon/osmium-fixed CF airway culture 24 hours after volume addition and with rotational mucus transport abolished. Note close apposition of mucus layer and the glycocalyx covering flattened cilia and the cell surface. (f) Light micrograph of freshly excised CF bronchus stained with Alcin blue periodic acid-Schiffs for mucus. As in the in vitro model, note close apposition (annealing) between secreted mucins and the cell surface (indicated by white arrow). NL, normal.

Although there have been a number of model systems designed to simulate cough clearance in artificial tubes, there have been no studies to evaluate the relationship between the mucus and the periciliary liquid layers, using cell culture models and confocal microscopy, in response to intraluminal air flow, as occurs during coughing.

ASL antimicrobial factors and the kinetics of mucus clearance. Clearance of bacteria from peripheral airways by mucus transport may require up to 6 hours. Since the number of bacteria can (under optimal conditions) double every 20 minutes, large increases in bacterial number on airway surfaces could occur during the 6-hour clearance period. However, it appears likely that antimicrobial substances (see Ganz, this Perspective series, ref. 34) in ASL suppress bacterial growth during mucus clearance. For example, the studies of Cole et al. have revealed that bacteria added to undiluted nasal surface liquid are initially "killed" for 3-6 hours by endogenous antimicrobial factors (35). These studies identified lactoferrin, lysozyme, and secretory leukoproteinase inhibitor as the major antimicrobial substances in ASLs; in contrast, defensins are present only in trace quantities. Cole et al. (35) also showed that the quantities of endogenous antimicrobial substances in ASL are sufficient to be "salt-insensitive"; that is, they were fully active in the salt concentrations (isotonic) measured in nasal ASL in these experiments. However, residual bacteria in these liquids acquired resistance to the ambient antimicrobial factors, and rapid bacterial growth recurred by 24 hours. This finding demonstrates an important kinetic interaction between antimicrobial substances in ASL and mucus clearance rates. Specifically, the antimicrobial substances can suppress bacterial growth for the short period (about 2-6 hours) that is normally required to clear inhaled bacteria from the airways by mucus transport. The prediction is that a failure to clear mucus would ultimately reveal the inability of chemical shields (antimicrobial substances) to chronically suppress bacterial growth in the lung.

\section{Genetic diseases associated with abnormal mucus clearance}

Primary ciliary dyskinesia: the importance of cough clearance. Patients with primary ciliary dyskinesia (PCD) exhibit recurrent middle ear infections, chronic airway infection, predominantly lower-lobe bronchiectasis, male sterility, and sometimes situs inversus. Patients with PCD exhibit a milder airway disease than do those with CF and typically live at least to middle age. This syndrome reflects defective ciliary function, with abnormalities in beat, stroke, or coordination, associated with genetically determined abnormalities of the structural/functional components of the ciliary shaft. The first of the genes associated with PCD has recently been cloned; it encodes an intermediate chain dynein (IC78) that appears important in assembling and coordinating the activities of several proteins, including the heavy chain dyneins of the ciliary shaft $(36,37)$.

The syndrome of PCD is informative with respect to many aspects of mucus clearance in lung defense. For example, measurements of mucociliary clearance, using external radionuclide tracers, have revealed virtually no basal, cilium-dependent mucus clearance in patients with PCD (38-40). However, studies of mucus clearance with inhaled radionuclide tracers show that cough-dependent mucus clearance is well preserved in PCD patients $(39,40)$. Thus, PCD patients may have a nearly normal rate of mucus clearance over time that is mediated solely by repetitive coughing. It appears likely that the retention of coughing as a backup 
mechanism for mucus clearance in PCD explains the milder pulmonary phenotype of PCD relative to CF. The presence of effective cough-dependent mucus clearance in PCD patients suggests that the volume of liquid on airway surfaces is normal or increased. Indeed, the few reports quantifying ion transport in airway tissues freshly excised from PCD patients or in nasal epithelia studied in vivo have revealed no abnormalities that might adversely affect the volume of ASL (41). Thus, the apparently normal ASL volume in PCD patients may both preserve the lubricant function of the PCL and provide sufficient hydration and height of mucus for effective cough-dependent clearance. Interestingly, agents such as UTP that increase the volume of liquid on airway surfaces can acutely increase the efficiency of cough clearance in PCD patients (39). Long-term studies, however, will be required to test the clinical benefit of such therapeutic strategies in these patients.

Cystic fibrosis: the importance of the periciliary liquid and mucus hypoxia. Although controversy still exists, it now seems likely that CF lung disease reflects chronic depletion of PCL volume rather than high salt concentrations in the CF ASL. This assertion rests upon evidence that ASL is isotonic both in the normal state (in humans and other large mammals) and in $\operatorname{CF}(5,6$, 42-44). Further, it is congruent with the findings that, under physiologic conditions, the rates of net epithelial $\mathrm{Na}^{+}$transport and isotonic volume absorption are higher in CF than in normal airway epithelia $(6,14,45$, 46). Consistent with accelerated volume absorption are reports of an increased number of $\mathrm{Na}^{+}-\mathrm{K}^{+}$-ATPase binding sites and activity, increased amiloride-sensitive and ouabain-sensitive $\mathrm{O}_{2}$ consumption in CF airway epithelia, and the early pathology of the non-infected CF lung $(33,41,47-49)$. Finally, recent data have directly connected reduced ASL volume, but not ion composition, with morphologic evidence of spontaneous airway disease in CF mice (50).

What is new in the area of CF pathogenesis is the detailed understanding of the relationships between volume depletion on airway surfaces and mechanisms for mucus transport. Volume depletion on airway surfaces could be associated with selective removal of liquid from the mucus layer, or could reflect depletion of both the mucus layer and the PCL. In vitro studies have revealed that volume depletion normally occurs sequentially, first from the mucus layer, and then from the PCL. Specifically, early volume depletion occurs from the mucus layer, until about $50 \%$ of volume is removed, after which the volume is removed from the PCL (51). Thus, the mucus layer can normally act as a reservoir for liquid, but in CF, where volume regulation on airway surfaces is severely perturbed, the capacity of the mucus layer to buffer the PCL volume may be exceeded.

Another important insight from these studies has been identification of the relative importance of depletion of the PCL in the pathogenesis of CF. Depletion of the PCL predicts adverse interactions between the mucus layer and the airway epithelial cell surface (Figure 4). Indeed, in vitro data indicate that depletion of the PCL allows the gel-forming mucins in the mucus layer, such as MUC5AC and MUC5B, to come in contact with the tethered mucins MUC1 and MUC4 on the cell surface (Figure 4). Similar findings have been made in analyses of frozen sections of freshly excised CF airways. Thus, it appears likely that the annealing that occurs between the carbohydrate side chains of the mobile mucins and cell surface mucins, which effectively glues mucins in mucus to cell surfaces (the "Velcro effect"), will abolish cough clearance. Hence, excessive ASL volume absorption constitutes a double hit on mucus clearance mechanisms. The loss of volume depletes the PCL, which removes the liquid in which cilia can extend and beat, thus inhibiting mucociliary clearance. It simultaneously compromises the PCL's lubricant function, allowing the mucus layer to adhere to cell surfaces and inhibiting cough clearance. This dual effect may account for the severe phenotype of CF lung disease, relative to PCD. Interestingly, the importance of tethered mucins in the pathogenesis of organ obstruction has been better documented in the gut, where the meconium ileus syndrome associated with the CF mouse intestine has been ameliorated by genetic ablation of the MUC1 mucin, which serves as the cell surface adhesive ligand for meconium in the intestine (52).

Recently, a link between mucus stasis and a predilection for Pseudomonas aeruginosa infection in CF has been revealed (33). In brief, despite the failure of mucus clearance, goblet cells continue to secrete, generating thick mucus plaques and plugs on airway surfaces. CF airway epithelia exhibit high rates of cellular $\mathrm{O}_{2}$ consumption to fuel raised $\mathrm{Na}^{+}$transport, which creates hypoxic zones in adherent mucus plaques near the cell surface. P. aeruginosa inhaled and deposited on the surfaces of mucus plaques "swim" into the mucus plaques and adapt to the hypoxic zones with alginate production and biofilm formation, setting the stage for chronic infection.

The initiating event in CF, ASL volume hyperabsorption, reflects two CF-specific defects in airway epithelial ion transport. Recent in vitro studies suggest that active $\mathrm{Na}^{+}$absorption by normal airway epithelia mediates ASL volume absorption under basal conditions and that normal airway epithelia can slow $\mathrm{Na}^{+}$absorption and induce $\mathrm{Cl}^{-}$secretion, when ASL volumes are depleted (51). In CF, there appear to be defects in both the $\mathrm{Na}^{+}$-absorptive and $\mathrm{Cl}^{-}$-secretory mechanisms for regulating ASL volume. Specifically, CF airway epithelia have an accelerated basal rate of $\mathrm{Na}^{+}$(and volume) absorption that reflects the absence of the tonic inhibitory effect of CFTR on the epithelial $\mathrm{Na}^{+}$channel $(\mathrm{ENaC})$ activity $(53,54)$. However, CF airway epithelia are also missing the capacity to add liquid back to airway surfaces when ASL volumes are depleted, due to the absence of CFTR functioning as a $\mathrm{Cl}^{-}$channel (55, 56). Thus, therapies directed both at slowing the abnormally raised volume absorption, e.g., $\mathrm{Na}^{+}$channel blockers, and at initiating $\mathrm{Cl}^{-}$channel activity, e.g., UTP-dependent activation of $\mathrm{Ca}^{2+}$-regulated $\mathrm{Cl}^{-}$channels, provide routes for the treatment of the primary defect of the CF airway epithelium $(20,57)$.

Pseudohypoaldosteronism: a surprise with respect to mucus clearance. Pseudohypoaldosteronism (PHA) is a clinical 
syndrome that reflects, at the genetic level, loss-of-function mutations in $\mathrm{ENaC}$ subunits (58). Characterization of the pulmonary phenotype of this syndrome became pertinent because of the contrasting predictions of the compositional (chemical shield) and volume (mucus clearance) hypotheses with respect to the effects of the loss of $\mathrm{Na}^{+}$channel function on ASL composition versus volume. Specifically, the compositional theory predicted that PHA patients would have raised ASL $\mathrm{NaCl}$ concentrations and inactivation of defensins, with resultant chronic airway infections. In contrast, the volume hypothesis predicted that PHA patients would have an excess of isotonic volume on airway surfaces, perhaps with a syndrome of airway obstruction.

Studies of PHA subjects showed that $\mathrm{Na}^{+}$transport is absent, at least as measured by the transepithelial potential difference technique (59). Analyses of both the upper (nasal) and lower (bronchial) airways revealed that the ASL is isotonic in PHA patients, as it is in healthy subjects, but that the volume of their ASL is greatly increased. These patients suffer intermittent airway obstruction and infection as young children, but after the age of 6 years, their lung function is normal, and they are free of chronic bacterial airway infections or bronchiectasis, consistent with normal lung defense. Studies of mucus clearance in these subjects with the external radiolabeled tracer technique (59) show that basal mucus clearance occurs at an astonishing rate, equaling or exceeding rates in normal subjects after acute exposures to $\beta$-agonists or purinergic agents. No comprehensive explanation for this observation is available, but, given the reservoir function of the mucus layer, extra liquid added to this mucus layer could account for a modest increase in mucus clearance rates. Indeed, recent in vitro studies have confirmed that liquid added to the ASL preferentially partitions into and swells the mucus layer, accelerating mucus transport (51). Whereas liquid addition probably improves mucus viscoelasticity, it likely also changes the characteristics of the PCL, perhaps decreasing its viscosity, which could also accelerate mucus transport.

Therefore, the mucus transport data from the PHA subjects have highlighted two principles. First, as long as mucus clearance is maintained, chronic airway infections do not occur, despite any postulated adverse effects of isotonic (high salt) ASL on antimicrobial activity. Second, expansion of ASL volume triggers a series of events in the ASL compartment that accelerates the rate of mucus transport.

\section{Future directions}

It has long been speculated that mucus clearance is important for airway defense, but only recently have important details of this system become available. For example, it has only been recently recognized that the nonspecific binding capacity of the carbohydrate side chains of mucins, coupled with the turbulent behavior of the mucus layer, mediates clearance of virtually all inhaled substances from the airways. Similarly, we have only recently recognized that the PCL not only promotes effective ciliary beat by providing a low vis- cosity solution but also serves an important role as a lubricant between the mobile and cell surface mucins. Finally, it has been primarily through the analysis of various genetic diseases of mucus transport that the importance of both the basal mucociliary clearance system and the back-up, cough-dependent system has become appreciated.

Many aspects of the airway defense system, however, remain to be elucidated. First, it is not yet clear at the physiologic level how this noninnervated epithelial system can regulate mucus transport rates over a more than threefold range. The preliminary observations that nucleotides are released by airway epithelia and interact with luminal purinoceptors provide the most tantalizing clues about the local regulation of mucus transport rates. Second, it is not yet clear how airway epithelia sense and regulate the volume of liquid on their surfaces. Although recent data indicate that regulation of ASL by airway epithelia involves the reciprocal regulation of active $\mathrm{Na}^{+}$absorption and $\mathrm{Cl}^{-}$secretion (51), it is not known whether the signals to the epithelium emanate from the ASL, or how such signals are transduced by the epithelium. Finally, whereas $\beta$-agonists and other agents that increase mucociliary clearance via regulation of ciliary beat frequency have become clinical mainstays, it now appears that other classes of drugs that affect the volume of ASL may be equally, or more, effective in many conditions and could be uniquely effective in specific diseases, such as CF. Thus, studies of airway function in health and disease have provided unique insights into how "wet-surface" epithelia can achieve a functional form of innate defense by unique adaptation of mechanical clearance mechanisms.

\section{Acknowledgments}

This work was supported by NIH grants HL34332, HL60280, R000046, and Cystic Fibrosis Foundation grant R026. The authors wish to thank their colleagues W. Bennett, C.W. Davis, H. Matsui, P.G. Noone, R. Pickles, S.H. Randell, M.J. Stutts, and R. Tarran, who have contributed to the knowledge underlying this perspective.

1. Kilburn, K.H. 1968. A hypothesis for pulmonary clearance and its implications. Am. Rev. Respir. Dis. 98:449-463.

2. Wine, J.J. 1999. The genesis of cystic fibrosis lung disease. J. Clin. Invest. 103:309-312.

3. Guggino, W.B. 1999. Cystic fibrosis and the salt controversy. Cell. 96:607-610.

4. Wanner, A., Salathe, M., and O'Riordan, T.G. 1996. Mucociliary clearance in the airways. Am. J. Respir. Crit. Care Med. 154:1868-1902.

5. Boucher, R.C. 1994. Human airway ion transport. Part 1. Am. J. Respir. Crit. Care Med. 150:271-281.

6. Boucher, R.C. 1994. Human airway ion transport. Part 2. Am. J. Respir. Crit. Care Med. 150:581-593.

7. Smith, J.J., Travis, S.M., Greenberg, E.P., and Welsh, M.J. 1996. Cystic fibrosis airway epithelia fail to kill bacteria because of abnormal airway surface fluid. Cell. 85:229-236.

8. Bals, R., Weiner, D.J., and Wilson, J.M. 1999. The innate immune system in cystic fibrosis lung disease. J. Clin. Invest. 103:303-307.

9. Goldman, M.J., et al. 1997. Human beta-defensin-1 is a salt-sensitive antibiotic that is inactivated in cystic fibrosis. Cell. 88:553-560.

10. Wine, J.J. 1997. A sensitive defense: salt and cystic fibrosis. Nat. Med. 3:494-495.

11. Boucher, R.C. 1999. Molecular insights into the physiology of the 'thin film' of airway surface liquid. J. Physiol. 516:631-638.

12. Sims, D.E., Westfall, J.A., Kiorpes, A.L., and Horne, M.M. 1991. Preser- 
vation of tracheal mucus by nonaqueous fixative. Biotech. Histochem. 66:173-180

13. Matsui, H., Randell, S.H., Peretti, S.W., Davis, C.W., and Boucher, R.C. 1998. Coordinated clearance of periciliary liquid and mucus from airway surfaces. J. Clin. Invest. 102:1125-1131.

14. Matsui, H., et al. 1998. Evidence for periciliary liquid layer depletion, not abnormal ion composition, in the pathogenesis of cystic fibrosis airways disease. Cell. 95:1005-1015.

15. Verdugo, P., Tam, P.Y., and Butler, J. 1983. Conformational structure of respiratory mucus studied by laser correlation spectroscopy. Biorbeology. 20:223-230.

16. Lamblin, G., et al. 2000. Human airway mucin glycosylation: a combinatory of carbohydrate determinants which vary in cystic fibrosis. BioLeXis. 1: 47 pp. http://www.biolexis.org.

17. Bernacki, S.H., et al. 1999. Mucin gene expression during differentiation of human airway epithelia in vitro. Muc 4 and muc5b are strongly induced. Am. J. Respir. Cell Mol. Biol. 20:595-604.

18. Lo-Guidice, J.M., et al. 1997. Mucins secreted by a transformed cell line derived from human tracheal gland cells. Biochem. J. 326:431-437.

19. Olivier, K.N., et al. 1996. Acute safety and effects on mucociliary clearance of aerosolized uridine 5 -triphosphate $+/$ - amiloride in normal human adults. Am. J. Respir. Crit. Care Med. 154:217-223.

20. Bennett, W.D., et al. 1996. Effect of uridine $5^{\prime}$-triphosphate plus amiloride on mucociliary clearance in adult cystic fibrosis. Am. J. Respir. Crit. Care Med. 153:1796-1801.

21. Jeffrey, P.K. 1998. The development of large and small airways. Am. J. Respir. Crit. Care Med. 157:S174-S180.

22. Knowles, M.R., Clarke, L.L., and Boucher, R.C. 1991. Activation by extracellular nucleotides of chloride secretion in the airway epithelia of patients with cystic fibrosis. N. Engl. J. Med. 325:533-538.

23. Davis, C.W., Dowell, M.L., Lethem, M.I., and Van Scott, M. 1992. Goblet cell degranulation in isolated canine tracheal epithelium: response to exogenous ATP, ADP, and adenosine. Am. J. Physiol. 262:C1313-C1323.

24. Lethem, M.I., et al. 1993. Nucleotide regulation of goblet cells in human airway epithelial explants: normal exocytosis in cystic fibrosis. Am. J. Respir. Cell Mol. Biol. 9:315-322.

25. Mall, M., et al. 2000. Inhibition of amiloride-sensitive epithelial $\mathrm{Na}(+)$ absorption by extracellular nucleotides in human normal and cystic fibrosis airways. Am. J. Respir. Cell Mol. Biol. 23:755-761.

26. Morse, D.M., Smullen, J.L., and Davis, C.W. 2001. Differential effects of UTP, ATP, and adenosine of ciliary activity of human nasal epithelial cells. Am. J. Physiol. 280:C1485-C1497.

27. Lazarowski, E.R., Boucher, R.C., and Harden, T.K. 2000. Constitutive release and extracellular interconversion of nucleotides. In Ecto-ATPases and related ectonucleotidases. L. Vanduffel and R. Lemmens, editors. Shaker Publishing BV. Maastricht, The Netherlands. 283-294.

28. Picher, M., and Boucher, R.C. 2000. Biochemical evidence for an ectoalkaline phosphodiesterase I in human airways. Am. J. Respir. Cell Mol. Biol. 23:255-261.

29. Lazarowski, E.R., Boucher, R.C., and Harden, T.K. 2000. Constitutive release of ATP and evidence for major contribution of ecto-nucleotide pyrophosphatase and nucleoside diphosphokinase to extracellular nucleotide concentrations. J. Biol. Chem. 275:31061-31068.

30. Picher, M., and Boucher, R.C. 2001. Metabolism of extracellular nucleotides in human airways by a multienzyme system. Drug Dev. Res. 52:66-75.

31. King, M. 1989. Mucus, mucociliary clearance and coughing. In Respiratory function in disease. D.V. Bates, editor. W.B. Saunders Co. Philadelphia, Pennsylvania, USA. 69-78.

32. Zahm, J.M., et al. 1989. The role of mucus sol phase in clearance by simulated cough. Biorheology. 26:747-752.

33. Worlitzsch, D., et al. 2002. Effects of reduced mucus oxygen concentration in airway Pseudomonas infections of cystic fibrosis patients. J. Clin. Invest. 109:317-325. DOI:10.1172/JCI200213870.

34. Ganz, T. 2002. Antimicrobial polypeptides in host defense of the respiratory tract. J. Clin. Invest. In press.

35. Cole, A.M., Dewan, P., and Ganz, T. 1999. Innate antimicrobial activity of nasal secretions. Infect. Immun. 67:3267-3275.

36. Pennarun, G., et al. 1999. Loss-of-function mutations in a human gene related to Chlamydomonas reinhardtii dynein IC78 result in primary ciliary dyskinesia. Am. J. Hum. Genet. 65:1508-1519.
37. Zariwala, M., et al. 2001. Germline mutations in an intermediate chain dynein cause primary ciliary dyskinesia. Am. J. Respir. Cell Mol. Biol. 25:577-583.

38. Camner, P., Mossberg, B., and Afzelius, B.A. 1983. Measurements of tracheobronchial clearance in patients with immotile-cilia syndrome and its value in differential diagnosis. Eur. J. Respir. Dis. Suppl. 64:57-63.

39. Noone, P.G., et al. 1999. Effect of aerosolized uridine-5' -triphosphate on airway clearance with cough in patients with primary ciliary dyskinesia. Am. J. Respir. Crit. Care Med. 160:144-149.

40. Noone, P.G., et al. 1996. Effects on cough clearance of aerosolized uridine- $5^{\prime}$-triphosphate $+/-$ amiloride in patients with primary ciliary dyskinesia. Am. J. Respir. Crit. Care Med. 153:A530. (Abstr.)

41. Knowles, M., Gatzy, J., and Boucher, R. 1981. Increased bioelectric potential difference across respiratory epithelia in cystic fibrosis. $N$. Engl. J. Med. 305:1489-1495.

42. Knowles, M.R., et al. 1997. Ion composition of airway surface liquid of patients with cystic fibrosis as compared to normal and disease-control subjects. J. Clin. Invest. 100:2588-2595.

43. Caldwell, R.A., et al. 2002. In vivo airway surface liquid $\mathrm{Cl}(-)$ analysis with solid-state electrodes. J. Gen. Physiol. 119:3-14.

44. Hull, J., Skinner, W., Robertson, C., and Phelan, P. 1998. Elemental content of airway surface liquid from infants with cystic fibrosis. Am.J. Respir. Crit. Care Med. 157:10-14.

45. Hirsh, A.J., Stutts, M.J., and Boucher, R.C. 2001. Bioelectric properties and ion flows of freshly-excised cystic fibrosis and normal nasal epithelium: a comparison to cultured airway epithelium. Pediatr. Pulmonol. Suppl. 19:238. (Abstr.)

46. Jiang, C., Finkbeiner, W.E., Widdicombe, J.H., McCray, P.B., Jr., and Miller, S.S. 1993. Altered fluid transport across airway epithelium in cystic fibrosis. Science. 262:424-427.

47. Peckham, D., Holland, E., Range, S., and Knox, A.J. 1997. $\mathrm{Na}^{+} / \mathrm{K}^{+}$ATPase in lower airway epithelium from cystic fibrosis and non-cystic-fibrosis lung. Biochem. Biophys. Res. Commun. 232:464-468.

48. Stutts, M.J., Knowles, M.R., Gatzy, J.T., and Boucher, R.C. 1986. Oxygen consumption and ouabain binding sites in cystic fibrosis nasal epithelium. Pediatr. Res. 20:1316-1320.

49. Zuelzer, W.W., and Newton, W.A., Jr. 1949. The pathogenesis of fibrocystic disease of the pancreas. A study of 36 cases with special reference to the pulmonary lesions. Pediatrics. 4:53-69.

50. Tarran, R., et al. 2001. The CF salt controversy: in vivo observations and therapeutic approaches. Mol. Cell. 8:149-158.

51. Tarran, R., Grubb, B.R., Gatzy, J.T., Davis, C.W., and Boucher, R.C. 2001. The relative roles of passive surface forces and active ion transport in the modulation of airway surface liquid volume and composition. $J$. Gen. Physiol. 118:223-236.

52. Parmley, R.R., and Gendler, S.J. 1998. Cystic fibrosis mice lacking Muc1 have reduced amounts of intestinal mucus. J. Clin. Invest. 102:1798-1806.

53. Stutts, M.J., et al. 1995. CFTR as a cAMP-dependent regulator of sodium channels. Science. 269:847-850.

54. Mall, M., Hipper, A., Greger, R., and Kunzelmann, K. 1996. Wild type but not delta F508 CFTR inhibits $\mathrm{Na}^{+}$conductance when coexpressed in Xenopus oocytes. FEBS Lett. 381:47-52.

55. Knowles, M.R., et al. 1983. Abnormal ion permeation through cystic fibrosis respiratory epithelium. Science. 221:1067-1070.

56. Bear, C.E., et al. 1992. Purification and functional reconstitution of the cystic fibrosis transmembrane conductance regulator (CFTR). Cell. 68:809-818.

57. Bennett, W.D., et al. 1994. Acute effect of aerosolized uridine $5^{\prime}$-triphosphate (UTP) + amiloride on mucociliary clearance in cystic fibrosis (CF). Am. J. Respir. Crit. Care Med. 149:A670. (Abstr.)

58. Chang, S.S., et al. 1996. Mutations in subunits of the epithelial sodium channel cause salt wasting with hyperkalaemic acidosis, pseudohypoaldosteronism type 1. Nat. Genet. 12:248-253.

59. Kerem, E., et al. 1999. Pulmonary epithelial sodium channel dysfunction and excess airway liquid in pseudohypoaldosteronism. N. Engl. J. Med. 341:156-162.

60. Blake, J.R., and Sleigh, M.A. 1974. Mechanics of ciliary locomotion. Biol. Rev. Camb. Philos. Soc. 49:85-125.

61. Fulford, G.R., and Blake, J.R. 1986. Muco-ciliary transport in the lung. J. Theor. Biol. 121:381-402. 